\title{
Instrumentos de investigación para el estudio del efecto de la música en el desarrollo de las destrezas lectoras
}

\author{
M. CARMen Fonseca-Mora, \\ MARÍA Gómez-Domínguez \\ Universidad de Huelva
}

Recibido: 6 septiembre 2013 / Aceptado: 1 noviembre 2014

ISSN: $1697-7467$

\begin{abstract}
RESUMEN: El aumento de estudios del efecto de la música en el desarrollo de las destrezas lectoras ha sido notable. Sin embargo, el panorama de resultados está fragmentado por tratarse de investigaciones multidisciplinares principalmente de lengua materna y con resultados diversos. En este artículo se revisan estudios de la última década para detallar los principales efectos encontrados y determinar los instrumentos de recogida de datos. Esto permite describir el estado actual de la cuestión y recomendar herramientas para futuros estudios sobre la relación entre música y desarrollo de destreza lectoras en una lengua extranjera.

Palabras clave: Música, destrezas lectoras, aprendizaje de lenguas, instrumentos de investigación
\end{abstract}

\section{Research Instruments for the Study of Musical Influence on Language Reading Skills}

\begin{abstract}
The increase in studies about the effect of music on the development of reading skills has been noticeable. However, the overview of results is fragmented due to multidisciplinary research mainly as regards $\mathrm{L} 1$ and with diverse results. This article reviews studies of the last decade to outline the main effects found and to determine the data collection instruments that have been used. It allows describing the current state of the issue and recommending tools for future studies on the relationship of music and reading skills in a foreign language.
\end{abstract}

Keywords: Music, reading skills, language learning, research instruments

\section{INTRODUCCIÓN}

La lectura es una habilidad esencial sobre la cual se construye el éxito en la educación formal. Se ha publicado mucho sobre el desarrollo de la capacidad lectora tanto en L1 como en L2 y de la transferencia del aprendizaje de la lectura de L1 a L2, sin embargo, los indicadores a nivel nacional e internacional siguen confirmando un déficit lector existente a nivel global. El Estudio Europeo de Competencia Lingüística (EECL, 2012) muestra que más de un 50\% de estudiantes europeos de 15-16 años de edad solo tiene nivel de principiante en LE. Al igual que en Europa, la evaluación nacional del progreso educativo de EEUU (NAEP, 2011) alerta de un porcentaje significativo de lectores con problemas y destaca la 
importancia de la intervención temprana, es decir, el énfasis de la enseñanza de las destrezas de descodificación en los primeros niveles educativos. Aún se necesitan métodos efectivos que puedan solucionar gran parte de los problemas lectores del alumnado.

Una de las alternativas para la enseñanza de lenguas que está en auge es la estimulación sonora-musical ya que melodías y ritmo facilitan el aprendizaje de lenguas maternas y extranjeras (Chobert y Besson, 2013; Toscano-Fuentes y Fonseca-Mora, 2012; Slevc y Miyake, 2006) y numerosos estudios avalan la relación entre música y desarrollo de destrezas lectoras (Tierney y Kraus, 2013a; Lessard y Bolduc, 2011; Standley, 2008; Butzlaff, 2000). Sin embargo, el panorama de resultados está fragmentado con resultados diversos fundamentalmente en L1. La investigación de la música y del desarrollo de las destrezas lectoras es objeto de estudio de disciplinas como la psicología, la neuropsicología, la educación tanto la general como la musical y la lingüística aplicada, con distintas herramientas de recogida de datos dada la complejidad del fenómeno a estudiar. Este número de herramientas y la dificultad per se de la investigación de sujetos en un aula influye también en el número de la muestra, por lo que a veces, estadísticamente los resultados han sido considerados como no concluyentes.

El objetivo de este artículo es revisar estudios existentes en L1 y LE para concretar resultados obtenidos y consensuar herramientas de recogida de datos para futuros estudios sobre la influencia musical en el desarrollo de las destrezas lectoras en LE.

\section{LA RELACión ENTRE LA MÚSICA Y EL PROCESO LECTOR}

Distintas investigaciones muestran que la aptitud musical se relaciona con la lectura (Toscano-Fuentes y Fonseca-Mora, 2012). Ambas comparten algunas destrezas auditivas como la discriminación melódica, rítmica y armónica y, en general, la combinación y segmentación de sonidos. Los procesos cognitivos para discriminar y asociar sonidos y tonos musicales con sus símbolos son similares a los procesos de descodificación grafema-fonema en la lectura. Hansen y Bernstorf (2002) relacionan la capacidad lectora de los niños con su capacidad para discriminar tonos o melodías y explican los componentes básicos de ambas: la conciencia fonológica, la conciencia fonémica, la identificación visual de palabras o de notas y los símbolos musicales, la conciencia ortográfica, la conciencia de los sistemas simbólicos, y la fluidez.

Los estudios sobre el efecto del programa musical en la conciencia fonológica (Herrera, Lorenzo, Defior, Fernández-Smith, y Costa-Giomi, 2011; Moreno, Marques, Santos, Santos, y Besson, 2009; Forgeard, Schlaug, Norton, Rosam, Iyengar, y Winner, 2008; Bolduc y Montésinos-Gelet, 2005; Gromko, 2005; Anvari, Trainor, Woodside, y Levy, 2002; Peynircioglu, Durgunoglu, Öney-Küssefoglu, 2002; Douglas y Willatts, 1994; Lamb y Gregory, 1993) concluyeron que los niños con mayor sensibilidad auditiva para discriminar sonidos consiguen mejores resultados en los tests de lectura. Sin embargo, Herrera et al. (2011) aportan que es el entrenamiento fonológico sin necesidad de apoyo musical el que mejora la conciencia fonológica y la velocidad de nombrar de los preescolares de su estudio mientras que los resultados de Gromko (2005) señalan que es la música la que afecta directamente a la capacidad de segmentar palabras en fonemas.

No hay acuerdo respecto al grado de relación entre percepción tonal, percepción rítmica y destrezas lectoras. Unos afirman que la percepción del tono es el único factor influyente en 
las conciencias fonológica y fonémica (Runfola, Etopio, Hamlen, y Rozendal, 2012; Tsang y Conrad, 2011; Bolduc y Montésinos-Gelet, 2005, y Lamb y Gregory, 1993), mientras que otros constatan que es la percepción rítmica (Tierney y Kraus, 2013b; Moritz, Yampolsky, Papadelis, Thomson, y Wolf, 2012; Forgeard et al., 2008; David, Wade-Woolley, Kirby, y Smithrim, 2007, y Douglas y Willatts, 1994).

Whalley y Hansen (2006) analizan la relación entre sensibilidad prosódica y desarrollo del proceso lector. Definen la prosodia como: "The phonological subsystem that encompasses the tempo, rhythm and stress of language" (p. 288). Los resultados muestran que la sensibilidad prosódica es fundamental para el desarrollo de la destreza lectora y que las destrezas prosódicas se relacionan con la comprensión lectora, la conciencia fonológica, la lectura de las palabras y la percepción del ritmo musical. Estos dos investigadores defienden que la comprensión lectora se puede interpretar como el producto de descodificación y comprensión auditiva, que da lugar al acceso léxico, y desde esta perspectiva, expresan que la prosodia es parte de la comprensión auditiva y, por lo tanto, se debe relacionar con la comprensión del texto.

Los estudios que abogan especialmente por el entrenamiento musical centrados en el desarrollo de destrezas lectoras tempranas (Register, Darrow, Standley, y Swedberg, 2007; Register, 2001; 2004) aseveran que estos programas tienen un efecto positivo en la adquisición de destrezas pre-lectoras y lectoras. Register et al. (2007) describen un programa musical multi-sensorial diseñado para mejorar las destrezas de descodificación de palabras, el aprendizaje de vocabulario y la comprensión de textos en niños sin y con problemas lectores. Los investigadores concluyeron que tanto los estudiantes sin problemas como los estudiantes con discapacidades en la lectura mejoraron los distintos componentes del proceso lector a excepción de la comprensión lectora.

Finalmente, es necesario mencionar las investigaciones con estudiantes diagnosticados con discapacidad en la lectura, tales como dislexia o deficiencias de habla y lenguaje, por lo que reciben una enseñanza especial (Huss, Verney, Fosker, Mead, y Goswami, 2011; Forgeard et al., 2008; Register et al., 2007; Register, 2001; Douglas y Willatts, 1994). De notable relevancia es que en todas ellas los programas musicales tienen un efecto positivo en el desarrollo de la destreza lectora de sus participantes. En el caso de niños disléxicos se comprueba que también tienen dificultades en las habilidades musicales de discriminación de tono y ritmo. Forgeard et al. (2008) demuestran que la enseñanza musical que se centra en la mejora del procesamiento tonal y rítmico puede ayudar a niños disléxicos en las destrezas auditivas necesarias para la lectura.

\section{MÉtodo}

\subsection{Objetivo y muestra}

El objetivo de este estudio es analizar investigaciones relacionadas con la percepción musical y el desarrollo de las destrezas lectoras para determinar los instrumentos de recogida de datos más usados según la tipología de estudio y el nivel educativo de los participantes con la finalidad de poder replicarlos en estudios de aprendizaje de lengua extranjera.

Para ello se han analizado 27 artículos internacionales publicados entre los años 20012013. Dichos estudios proceden de áreas diversas como lingüística aplicada (enseñanza de 
lenguas, fonética), educación, musicología (musicoterapia), y psicología (neuropsicología). Las publicaciones científicas de este corpus han sido escogidas teniendo en cuenta los siguientes criterios de selección:

- Por temática: estudios sobre uso de melodías y ritmo para mejorar el proceso lector.

- Por impacto: estudios publicados en revistas de impacto por lo que han pasado los filtros de evaluación de expertos.

- Por tipología: estudios de carácter experimental, cuasi-experimental y de correlación.

Este corpus contiene artículos indexados en ISI "Web Of Knowledge", ERIC, JSTOR y "Academic Search Premier". La admisión de estas revistas de impacto y ranking en las bases de datos antes citadas supone que han sido evaluadas positivamente por su calidad de los procesos editoriales, su buen funcionamiento de los indicadores de difusión y visibilidad, sus indicadores de impacto y uso y, finalmente, sus indicadores sobre la calidad del contenido.

\subsection{Sistema de criterio de análisis}

El análisis ha tenido en cuenta las siguientes variables:

- Tipología del estudio: correlacional, cuasi-experimental y experimental.

- Características de los participantes: número de participantes, nivel educativo, clasificación educacional (estudiantes sin y con discapacidad en el aprendizaje de la lectura) y nacionalidad.

- Lenguas en el estudio: lengua materna y/o segundas lenguas o lenguas extranjeras.

- Instrumentos de recogida de datos: tests estandarizados, pruebas diseñadas por los investigadores del estudio.

\subsection{Análisis de los estudios}

Las investigaciones contrastadas son once estudios de correlación, ocho cuasi-experimentales y ocho experimentales. Los estudios de correlación comparan componentes del proceso lector con aptitud musical pero no incluyen ningún tipo de programa de intervención por lo que no se establece una relación causa-efecto entre música y destrezas lectoras como en el caso de los experimentales. Los experimentales y los cuasi-experimentales contienen uno o varios pre-tests, un programa de intervención sonoro-musical especialmente diseñado para el aprendizaje de la lectura o un programa de intervención estrictamente musical o convencional, y uno o varios post-tests para así verificar el progreso del alumnado. La limitación de los cuasi-experimentales es que los participantes no son seleccionados aleatoriamente como en los experimentales, lo que es considerado como condición esencial para establecer una relación causal entre las diferentes variables (Lessard y Bolduc, 2011:116).

\subsubsection{Estudios de correlación}

La tabla 1 muestra los estudios de correlación seleccionados y las distintas pruebas usadas. Se indica también el nivel educativo, el número de los participantes y la frecuencia de uso de cada prueba con relación a los demás estudios correlacionales. 
Tabla 1. Instrumentos de recogida de datos, muestra y nivel educativo en estudios de correlación

\begin{tabular}{|c|c|c|c|c|c|c|c|c|}
\hline & \multicolumn{8}{|c|}{ Instrumentos de recogida de datos } \\
\hline & $\begin{array}{l}\text { Lectura } \\
(90,9 \%)\end{array}$ & $\begin{array}{c}\text { Vocabulario } \\
(27,7 \%)\end{array}$ & $\begin{array}{c}\text { Conciencia } \\
\text { fonológica/ } \\
\text { fonémica } \\
(100 \%)\end{array}$ & $\begin{array}{c}\text { Velocidad } \\
\text { de } \\
\text { nombrar } \\
(18,2 \%)\end{array}$ & $\begin{array}{c}\mathrm{CI} / \\
\text { memoria } \\
\text { verbal } \\
(54,5 \%)\end{array}$ & $\begin{array}{c}\text { Aptitud } \\
\text { musical } \\
y \\
\text { auditiva } \\
(90,9 \%)\end{array}$ & $\begin{array}{c}\text { Prosodia } \\
(9 \%)\end{array}$ & $\begin{array}{c}\text { Cuestionario } \\
\text { socio- } \\
\text { económico/ } \\
\text { cultural } \\
(27,7 \%)\end{array}$ \\
\hline $\begin{array}{l}\text { Autores } \\
\text { y muestra }\end{array}$ & \multicolumn{8}{|c|}{ Preescolar } \\
\hline $\begin{array}{c}\text { Anvari } \\
\text { et al. }(2002) \\
n=100\end{array}$ & $\checkmark$ & $\checkmark$ & $\checkmark$ & & $\checkmark$ & $\checkmark$ & & \\
\hline $\begin{array}{c}\text { Bolduc, y } \\
\text { Montésinos- } \\
\text { Gelet (2005) } \\
\mathrm{n}=13\end{array}$ & & & $\checkmark$ & & & $\checkmark$ & & \\
\hline $\begin{array}{c}\text { Peynircioglu } \\
\text { et al. (2002) } \\
\mathrm{n}=72(2 \\
\text { estudios) }\end{array}$ & $\checkmark$ & & $\checkmark$ & & & $\checkmark$ & & \\
\hline $\begin{array}{l}\text { Autores } \\
\text { y muestra }\end{array}$ & \multicolumn{6}{|c|}{ Primaria } & & \\
\hline $\begin{array}{c}\text { Banai y } \\
\text { Ahissar } \\
(2013) \\
\mathrm{n}=156 \\
\end{array}$ & $\checkmark$ & & $\checkmark$ & & $\checkmark$ & $\checkmark$ & & \\
\hline $\begin{array}{c}\text { Corrigal y } \\
\text { Trainor } \\
(2011) \\
n=46 \\
\end{array}$ & $\checkmark$ & & $\checkmark$ & & $\checkmark$ & & & $\checkmark$ \\
\hline $\begin{array}{c}\text { David } \\
\text { et al. }(2007) \\
\mathrm{n}=53\end{array}$ & $\checkmark$ & & $\checkmark$ & $\checkmark$ & & $\checkmark$ & & \\
\hline $\begin{array}{c}\text { Forgeard et } \\
\text { al. (2008) } \\
\mathrm{n}=100(4 \\
\text { estudios) }\end{array}$ & $\checkmark$ & & $\checkmark$ & & $\checkmark$ & $\checkmark$ & & $\checkmark$ \\
\hline $\begin{array}{c}\text { Tsang y } \\
\text { Conrad } \\
(2011) \\
n=69\end{array}$ & $\checkmark$ & $\checkmark$ & $\checkmark$ & & & $\checkmark$ & & $\checkmark$ \\
\hline $\begin{array}{c}\text { Whalley y } \\
\text { Hansen } \\
(2006) \\
n=81 \\
\end{array}$ & $\checkmark$ & & $\checkmark$ & & & $\checkmark$ & $\checkmark$ & \\
\hline $\begin{array}{c}\text { Zuk et al. } \\
(2013) \\
n=43\end{array}$ & $\checkmark$ & & $\checkmark$ & $\checkmark$ & $\checkmark$ & $\checkmark$ & & \\
\hline $\begin{array}{l}\text { Autores } \\
\text { y muestra }\end{array}$ & \multicolumn{8}{|c|}{ Primaria y secundaria } \\
\hline $\begin{array}{c}\text { Huss et al. } \\
(2011) \\
n=64\end{array}$ & $\checkmark$ & $\checkmark$ & $\checkmark$ & & $\checkmark$ & $\checkmark$ & & \\
\hline
\end{tabular}


La población participante oscila entre los 13 del estudio piloto de Bolduc y MontésinosGelet (2005) y los 156 de Banai y Ahissar (2013), siendo la media de 72,4 estudiantes. Solo los participantes del estudio de Huss et al. (2011) y algunos de los participantes de Forgeard et al. (2008) son disléxicos. Un 63,6 \% se refiere a estudiantes de primaria mientras que un $27,3 \%$ se refiere a preescolar y un $9 \%$ a estudiantes de primaria y secundaria (8-13 años). La mayoría de los participantes son de habla inglesa (anglo-canadienses, ingleses, estadounidenses y australianos) si bien también se encuentran estudios con niños francocanadienses (Bolduc y Montésinos-Gelet. 2005), turcos (Peynircioglu et al. 2002), brasileños (Zuk, Andrade, Andrade, Gardiner, y Gaab, 2013) y hebreos-israelíes (Banai y Ahissar, 2013). Casi todos son estudios de lengua materna, a excepción del de David et al. (2007) donde el alumnado de origen caucásico es residente en Canadá.

El único estudio que no incluye prueba de lectura es el de Bolduc y Montésinos-Gelet (2005) que solo evaluó la conciencia fonológica de niños preescolares. La prueba Phonological Awareness Test (PAT) es la más usada para evaluar la conciencia fonológica, si bien para evaluar esta destreza Anvari et al. (2002) y Corrigal y Trainor (2011) utilizaron los tests Auditory Analytic Skills y Standarized Auditory Perception Test, mientras que Forgeard et al. (2008) y Tsang y Conrad (2013) utilizaron el Test of Auditory Analysis Skills (TAAT). Las pruebas de conciencia fonológica son mayoritariamente de identificación de sílabas y sonidos iniciales y finales, y tareas de combinación y omisión de fonemas.

Se observa también una gran variedad de pruebas estandarizadas de lectura, siendo las más comunes los tests Wide Range Achievement Test-3 (WRAT-3), Neale Analysis of Reading Ability-Revised, la escala de habilidades lectoras Bristish Abilities Scale (BAS), y la conocida prueba Woodcock Reading Mastery Tests-Revised (WRMTR). Además, los estudios de Anvari et al. (2002), Huss et al. (2011) y Tsang y Conrad (2011) han completado la habilidad lingüística con las pruebas de vocabulario Peabody Picture Vocabulary Test-Revised y 4th Edition (PPVT-R; PPVT-4) y la escala Bristish Picture Vocabulary Scale (BPVS), mientras que David et al. (2007) y Zuk et al. (2013) utilizaron pruebas de nombramiento de estímulos visuales como colores, números o imágenes.

La prueba neuropsicológica de inteligencia general utilizada en un 54,5\% de los estudios es el test Wechsler Intelligence Scale for Children (WISC) en sus distintas versiones adaptadas a las edades de los participantes, con especial énfasis en la tarea de "dígitos" (Digit Span) de memoria de trabajo verbal. Además, Zuk et al., (2013) utilizaron también tareas de memoria visual extraídas del test Cognitive Linguistic Protocol. Mientras que Corrigal y Trainor (2011) es el único estudio que no incluye un test de aptitud musical que evalúe la percepción melódica, rítmica y armónica, Whalley y Hansen (2006) se diferencia de los demás por incluir pruebas de sensibilidad prosódica: The DEEdee Task y dos tareas del test Profiling Elements of Prosodic Systems - Children test. Por último, algunos estudios como Corrigal y Trainor (2011) y Tsang y Conrad (2011) recogen información demográfica sobre la familia, datos del nivel educativo de las madres y datos de formación musical de padres e hijos. Corrigal y Trainor (2011), además, recoge datos de los hábitos lectores y musicales de ambos.

\subsubsection{Estudios cuasi-experimentales}

La tabla 2 muestra los estudios cuasi-experimentales seleccionados y las distintas pruebas usadas. Se indica también el nivel educativo, el número de los participantes y la frecuencia de uso de cada prueba en relación a los demás estudios cuasi-experimentales. 
Tabla 2. Instrumentos de recogida de datos, muestra y nivel educativo en estudios cuasi-experimentales.

\begin{tabular}{|c|c|c|c|c|c|c|}
\hline & \multicolumn{6}{|c|}{ Instrumentos de recogida de datos } \\
\hline & $\begin{array}{c}\text { Lectura/ } \\
\text { lecto-escritura/ } \\
\text { lenguaje oral } \\
(100 \%)\end{array}$ & $\begin{array}{c}\text { Vocabulario } \\
(50 \%)\end{array}$ & $\begin{array}{c}\text { Conciencia } \\
\text { fonológica/ } \\
\text { fonémica/ } \\
\text { velocidad de } \\
\text { nombrar } \\
(75 \%)\end{array}$ & $\begin{array}{c}\text { Aptitud } \\
\text { musical y } \\
\text { auditiva } \\
(37,5 \%)\end{array}$ & $\begin{array}{c}\mathrm{CI} / \\
\text { memoria de } \\
\text { trabajo verbal } \\
(25 \%)\end{array}$ & $\begin{array}{l}\text { Cuestionario } \\
\text { socio- } \\
\text { económico } \\
(12,5 \%)\end{array}$ \\
\hline $\begin{array}{c}\text { Autores y } \\
\text { muestra }\end{array}$ & \multicolumn{6}{|l|}{ Preescolar } \\
\hline $\begin{array}{c}\text { Gromko, } \\
(2005) \\
\mathrm{n}=103 \\
\end{array}$ & $\checkmark$ & & $\checkmark$ & & & \\
\hline $\begin{array}{c}\text { Register, } \\
(2001) \\
n=50\end{array}$ & $\checkmark$ & & & & & \\
\hline $\begin{array}{c}\text { Autores y } \\
\text { muestra }\end{array}$ & \multicolumn{6}{|c|}{ Preescolar y primaria } \\
\hline $\begin{array}{c}\text { Fisher, } \\
(2001) \\
\mathrm{n}=80\end{array}$ & $\checkmark$ & & $\checkmark$ & & & \\
\hline $\begin{array}{l}\text { Moritz et } \\
\text { al. (2012) } \\
\mathrm{n}=42(2 \\
\text { estudios) }\end{array}$ & $\checkmark$ & $\checkmark$ & $\checkmark$ & $\checkmark$ & $\checkmark$ & \\
\hline $\begin{array}{c}\text { Register, } \\
(2004) \\
\mathrm{n}=86 \\
\end{array}$ & $\checkmark$ & & $\checkmark$ & & & $\checkmark$ \\
\hline $\begin{array}{c}\text { Autores y } \\
\text { muestra }\end{array}$ & \multicolumn{6}{|l|}{ Primaria } \\
\hline $\begin{array}{c}\text { Bhide et } \\
\text { al. }(2013) \\
n=19\end{array}$ & $\checkmark$ & $\checkmark$ & $\checkmark$ & $\checkmark$ & & \\
\hline $\begin{array}{c}\text { Moreno et } \\
\text { al. (2009) } \\
\mathrm{n}=32\end{array}$ & $\checkmark$ & & $\checkmark$ & $\checkmark$ & $\checkmark$ & \\
\hline $\begin{array}{l}\text { Register } \\
\text { et al. } \\
(2007) \\
n=41\end{array}$ & $\checkmark$ & $\checkmark$ & & & & \\
\hline
\end{tabular}

El número de participantes oscila entre los 19 del estudio de Bhide, Power, y Goswami (2013) y los 103 de Gromko (2005) siendo la media de 56,6 estudiantes. Estos estudios se centran en el desarrollo de las destrezas lectoras en lengua materna tanto en preescolar (25\%) como en cursos entre preescolar y primaria (37,5\%) y en los primeros cursos de primaria $(37,5 \%)$ principalmente en estudiantes estadounidenses, aunque también se encuentran participantes portugueses (Moreno et al. 2009), hispanos (Fisher, 2001) y hebreos-israelíes (Bhide et al., 2013). De notable importancia es que el estudio de Fisher (2001) es el único que incluye estudiantes que reciben educación bilingüe, en este caso de español-inglés. Un reducido número de estudios afecta también a alumnado con alguna discapacidad en la lectura (Bhide et al., 2013; Register, 2001; Register et al., 2007).

La tabla 2 recoge una variedad de pruebas estandarizadas que van desde pruebas de lectura adaptadas a los distintos niveles y lenguas (en 100\% de los estudios), a las de vo- 
cabulario (en $50 \%$ de los estudios) y conciencia fonológica (en $75 \%$ de los estudios) que a veces ya están incluidas en las estandarizadas de lectura, y finalmente de aptitud musical $(37,5 \%)$ e inteligencia general $(25 \%)$.

Gromko (2005) y Register (2004) utilizaron la prueba de alfabetización y conciencia fonológica Dynamic Indicators of Basic Early Literacy Skills (DIBELS) mientras los demás estudios incluyen tests estandarizados de lectura: Test of Word Reading Efficiency (TOWRE), Woodcock Reading Mastery Tests-Revised (WRMT-R), Developmental Reading Assessment (DRA), Test of Early Reading Ability-3rd Edition (TERA-3) y Gates-MacGinitie Reading Test, 4th Edition (GMRT-4), así como otros de conciencia fonológica: Phonological Awareness Test (PAT) y Comprehensive Test of Phonological Processing (CTOPP). Los tests de vocabulario usados son el de Peabody Picture Vocabulary Test III (PPVT-III) y el test British Picture Vocabulary Scale-II (BPVS II). Moritz et al., (2012) es el único que incluye un test estandarizado de aptitud musical (Musical Aptitude Test, MAT), mientras que Bhide et al. (2013) y Moreno et al. (2009) usan tareas de discriminación tonal en melodías y tareas de percepción auditiva creadas por ellos. Solo dos estudios incluyen un test de inteligencia general. Moreno et al. (2009) utilizaron diez tareas de una versión portuguesa del test Wechsler Intelligence Scale for Children, 3rd Edition (WISC-III). Además, para medir la memoria verbal a corto plazo y la memoria de trabajo se administró la tarea de "dígitos" de este mismo test (Digit Span). Por otro lado, Moritz et al. (2012) utilizaron el test de inteligencia verbal y no verbal Kaufman Brief Intelligence Test (K-BIT). Finalmente, solo Register (2004) recoge datos socio-económicos sobre la familia a partir de un cuestionario.

\subsubsection{Estudios experimentales}

La tabla 3 indica los estudios experimentales seleccionados, las distintas pruebas usadas, el nivel educativo, el número de los participantes y la frecuencia de uso de cada prueba en relación a los demás estudios experimentales.

Tabla 3. Instrumentos de recogida de datos, muestra y nivel educativo en estudios experimentales.

\begin{tabular}{|c|c|c|c|c|c|c|c|c|}
\cline { 2 - 7 } & \multicolumn{9}{c|}{ Instrumentos de recogida de datos } \\
\cline { 2 - 7 } & $\begin{array}{c}\text { Lectura } \\
(50 \%)\end{array}$ & $\begin{array}{c}\text { Vocabulario } \\
(12,5 \%)\end{array}$ & $\begin{array}{c}\text { Conciencia } \\
\text { fonológica/ } \\
\text { fonémica } \\
(75 \%)\end{array}$ & $\begin{array}{c}\text { Velocidad } \\
\text { de nombrar } \\
(12,5 \%)\end{array}$ & $\begin{array}{c}\text { Aptitud } \\
\text { musical } \\
(37,5 \%)\end{array}$ & $\begin{array}{c}\text { CI/ } \\
\text { memoria } \\
\text { verbal, } \\
(62,5 \%)\end{array}$ & $\begin{array}{c}\text { Cuestionario } \\
\text { socio- } \\
\text { económico/ } \\
\text { cultural } \\
(25 \%)\end{array}$ \\
\hline $\begin{array}{c}\text { Autores y } \\
\text { muestra }\end{array}$ & Preescolar & & $\checkmark$ & & $\checkmark$ & & \\
\hline $\begin{array}{c}\text { Bolduc, } \\
(2009) \\
\text { n=105 }\end{array}$ & & & $\checkmark$ & & $\checkmark$ & & $\checkmark$ \\
\hline $\begin{array}{c}\text { Carmon } \\
\text { et al. } \\
(2008) \\
n=150\end{array}$ & $\checkmark$ & & & & & & & \\
\hline
\end{tabular}




\begin{tabular}{|c|c|c|c|c|c|c|c|}
\hline $\begin{array}{c}\text { Degé y } \\
\text { Schwarzer } \\
,(2011) \\
n=41\end{array}$ & & & $\checkmark$ & & & $\checkmark$ & $\sqrt{ }$ \\
\hline $\begin{array}{c}\text { Herrera } \\
\text { et al. } \\
(2011) \\
\mathrm{n}=97\end{array}$ & $\checkmark$ & $\checkmark$ & $\checkmark$ & $\checkmark$ & & $\checkmark$ & \\
\hline $\begin{array}{c}\text { Moreno et } \\
\text { al. }(2011) \\
\mathrm{n}=60\end{array}$ & & & $\bar{V}$ & & & $\checkmark$ & $\bar{\gamma}$ \\
\hline $\begin{array}{c}\text { Runfola et } \\
\text { al. (2012) } \\
n=165\end{array}$ & $\checkmark$ & & & & $\checkmark$ & & \\
\hline $\begin{array}{c}\text { Autores y } \\
\text { muestra }\end{array}$ & Primaria & & & & & & \\
\hline $\begin{array}{c}\text { Cogo- } \\
\text { Moreira et } \\
\text { al. }(2013) \\
n=235\end{array}$ & & & $\checkmark$ & & & $\checkmark$ & \\
\hline $\begin{array}{c}\text { Autores y } \\
\text { muestra }\end{array}$ & Secunda & & & & & & \\
\hline $\begin{array}{c}\text { Taub y } \\
\text { Lazarus, } \\
(2013) \\
\mathrm{n}=280\end{array}$ & $\sqrt{\checkmark}$ & & & & & & \\
\hline
\end{tabular}

El número de participantes oscila entre los 41 de Degé y Schwarzer (2011) y los 280 de Taub y Lazarus (2013), siendo la media una población de 142. La mayoría de los estudios son de preescolar (75\%), a excepción de dos: el de Cogo-Moreira et al. (2013) en el que participaron estudiantes de $4^{\circ}$ de primaria y el de Taub y Lazarus (2013) que es el único en todo el corpus con estudiantes de secundaria (edad media 15 años). Estos estudios se centran en el desarrollo de las destrezas lectoras en lengua materna y recogen datos de participantes de siete lenguas distintas, mostrando una población de franco-canadienses (Bolduc, 2009), canadienses (Moreno et al., 2011), israelíes (Carmon et al., 2008), brasileños (Cogo-Moreira et al., 2013), alemanes (Degé y Schwarzer, 2011), estadounidenses (Forgeard et al., 2008) y españoles (Herrera et al., 2011). Digno de mención es el estudio de Herrera et al. (2011) ya que es el único en el que parte de su población son alumnos (hablantes de Tamazight) que estudian una segunda lengua, en este caso, el español. Además, los participantes del estudio de Runfola et al. (2012) tienen orígenes étnicos muy variados: un $66 \%$ son de origen caucásico mientras que un $19 \%$ son afro-americanos y un $8 \%$ hispanos; el $7 \%$ restante corresponde a estudiantes de otros orígenes étnicos.

Las distintas pruebas han sido administradas antes y después del programa de intervención musical. En la tabla 3 se observa un aumento de pruebas estandarizadas en todas las destrezas. La prueba de conciencia fonológica Phonological Awareness Test (PAT) es la más frecuente, también se incluyen las pruebas de Woodcook-Johnson III, Test of Cognitive Abilities Sound awareness. En algunos casos las pruebas de conciencia fonológica ya están incluidas en los tests de lecto-escritura por estar adaptadas al nivel preescolar o los primeros cursos de primaria. Entre las pruebas de lectura estandarizadas se observan las siguientes: 
Woodcock-Johnson Tests of Achievement-III, Informal Reading Inventory (IRI), Test of Language Development: Primary, 3rd Edition (TOLD: P3) y Test de Análisis Lectoescritor (TALE). El test de inteligencia general de Wechsler (WISC) en sus distintas versiones adaptadas a las distintas lenguas y edades es el más frecuente. Varios estudios incluyen la tarea de "dígitos" del test de Wechsler para conocer el efecto del entrenamiento musical en la memoria verbal de trabajo porque como Ho, Cheung y Chan (2003) establecieron, el entrenamiento musical afecta a la memoria verbal y no a la memoria visual de los niños. Solo tres estudios (Bolduc, 2009; Carmon et al. 2008; Runfola et al. 2012) utilizaron tests de aptitud musical: una versión adaptada del test Primary Measures of Music Audiation y del test Music Ability Test, y los tests Singing Voice Development Measure (SVDM) y Test of Early Audiation Achievement (T-EAA). Finalmente, un 25\% de los estudios recaban datos también con un cuestionario socio-cultural sobre la familia.

\section{Discusión}

La mayoría de los estudios son con estudiantes de infantil o de primaria, reduciéndose a dos los que explican algunos resultados en secundaria. Se estudia principalmente el efecto de la música, melodías y/o ritmo en las destrezas auditivas y de descodificación, en las conciencias fonológica y fonémica, en la memoria verbal, su conexión con la discriminación tonal y con otros elementos suprasegmentales de la prosodia que afectan el acceso léxico y la comprensión lectora. En algunas ocasiones las publicaciones analizan terapias musicales para niños con algún tipo de discapacidad, especialmente disléxicos.

La temporalización de los estudios ha sido un elemento de dificultad para interpretar en este meta-análisis. Mientras la mayoría de los estudios de correlación detallan la duración de las sesiones de administración de las pruebas que se llevaron a cabo en días o en semanas -a excepción del estudio longitudinal de David et al. (2007) que tuvo una duración de 5 años-, los estudios experimentales y cuasi-experimentales que especifican la duración de sus intervenciones abarcan una amplia gama de períodos que van desde 4 semanas hasta 2 y 3 años.

En los estudios correlacionales se identifican cuatro baterías de instrumentos claves para estudiar la relación entre la música y las destrezas lectoras: tests de lecto-escritura y tests de aptitud musical (usados ambos en un 90,9\% de los estudios), tests de conciencia fonológica (usados en todos los estudios) y tests de inteligencia y memoria verbal (usados en un $54,5 \%$ ). Los estudios de naturaleza experimental o cuasi-experimental intentan confirmar la relación causal entre la música y las destrezas lectoras a partir de la puesta en práctica en el aula de programas de intervención fonológicos-musicales especialmente diseñados para el aprendizaje de la lectura o en ocasiones de programas estrictamente musicales llevados a cabo en centros escolares. Debido a que cada estudio ha utilizado su programa de intervención musical y la descripción que ofrecen no es completa, no se puede concluir qué tipo de intervención es la más efectiva. En los cuasi-experimentales se observan por frecuencia de uso en los estudios tres baterías de instrumentos claves para analizar la relación causal entre la música y las destrezas lectoras: tests de lecto-escritura (100\%), tests de conciencia fonológica $(75 \%)$ y tests de vocabulario $(50 \%)$ mientras que en los experimentales se de- 
tectan tres tipos de pruebas claves: una batería de pruebas de conciencia fonológica $(75 \%)$; una batería de instrumentos de lecto-escritura (50\%), y una serie de tests de inteligencia general y memoria verbal $(62,5 \%)$.

La capacidad lectora de los niños está también influenciada por los niveles educativos parentales, especialmente de la madre, y los hábitos lectores familiares así como las distintas lenguas que se hablen en el hogar (Phillips, Norris, y Anderson, 2008; Ramón y Sánchez, 2009). Existe consenso con respecto a que el hábito familiar de leerles libros a los niños desde edades tempranas incide posteriormente en el buen desarrollo de la capacidad lectora del niño (Foy y Mann, 2003; Karrass y Braungart-Rieker, 2005; Lane y Wright, 2007; Phillips et al., 2008; Ramón y Sánchez, 2009). Por ello, el 22,2 \% de los estudios de este meta-análisis aportan un elemento relevante de visión al recabar también datos mediante un cuestionario socio-cultural y económico.

Los estudios de este meta-análisis reflejan el efecto positivo de la instrucción musical en la enseñanza de habilidades pre-lectoras de niños preescolares y muestran una mejora en las habilidades lectoras de niños de primaria sin problemas en la lectura y de niños disléxicos. Sin embargo, solo un 7,4\% refleja estudios relacionados con el aprendizaje de la lectura en una L2 (Herrera et al., 2011; Fisher, 2001). Las hipótesis de transferencia entre L1 y L2 definen que el aprendizaje de la lectura en L2 se basa en los procesos cognitivos adquiridos en L1, si bien el nivel de competencia comunicativa en cualquier lengua es una variable determinante. Sería interesante administrar una prueba de lecto-escritura que contrastara las destrezas básicas del alumnado en ambas lenguas con el objeto posterior de subsanar las deficiencias detectadas.

Por otro lado, en el caso de estudiantes españoles aprendiendo inglés como LE, las dificultades del aprendizaje van estrechamente unidas a la opacidad de la lengua meta con respecto a las correspondencias entre su pronunciación y escritura y a sus diferencias isocrónicas. El inglés tiene un alfabeto de 26 letras, 44 fonemas, y éstos aproximadamente 70 grafemas o formas distintas de escribirse, mientras que el español es una lengua transparente. Contrastan también con respecto a su ritmo o isocronía. El inglés es una lengua isoacentual, la duración entre dos sílabas acentuadas es igual, mientras que el español es isosilábica, es decir, la duración de cada sílaba es la misma, lo que provoca un ritmo distinto en la lectura que también se ha de aprender. Los pocos estudios sobre L2 encontrados para este metaanálisis apuntan a que un programa de intervención fonológico-musical incide también en el aprendizaje del proceso lector de estudiantes de una segunda lengua. Sin embargo, los estudios de este meta-análisis no incluyen pruebas de lecto-escritura administradas en ambas lenguas ni describen si en sus programas de intervención se trabajan estos elementos isocrónicos.

\section{Conclusiones}

Tal como se ha comentado con anterioridad, de notable importancia es que la mayoría de los estudios que conforman este meta-análisis son estudios monolingües. Por lo tanto, serían necesarias futuras investigaciones sobre la relación entre música y destrezas lectoras en una LE ya que los datos indican una alternativa efectiva de enseñanza de las habilidades lectoras. Dada la complejidad de variables que afectan al proceso lector, más de $51,9 \%$ de 
los estudios administra 4 o 5 pruebas. La mayoría de éstas requiere un tiempo de administración de entre 15-40 minutos aproximadamente lo que afecta a la organización y rutina docente en los centros escolares. Otro elemento de dificultad es que ciertas pruebas, como las de inteligencia general y memoria verbal, requieren ser administradas por profesionales del ámbito de la psicología por lo que podría ser beneficioso un equipo multidisciplinar.

A la luz de de los estudios revisados, la investigación de la naturaleza causal entre música y desarrollo de las destrezas lectoras en una LE puede contemplar la siguiente batería de instrumentos estandarizados con el objeto de lograr una visión completa: prueba de lecto-escritura adaptada a L1 y LE, con pruebas de conciencia fonológica, fonémica y vocabulario adaptadas al nivel de los participantes; prueba de inteligencia general y memoria verbal; prueba de aptitud musical, y cuestionario socio-cultural sobre el nivel educativo de los padres, los hábitos músico-lectores familiares y las lenguas que se hablan en el hogar. No obstante, la decisión final de los instrumentos a usar depende del objetivo central del estudio.

\section{Agradecimientos}

Estudio financiado por el Ministerio de Ciencia e Innovación de España: FFI201015738 "Percepción musical y destrezas lectoras en el aprendizaje de una lengua extranjera"

\section{REFERENCIAS}

Los estudios del corpus están incluidos.

Anvari, S.H., Trainor, L.J., Woodside, J., y Levy, B.A. (2002). "Relations among musical skills, phonological processing, and early reading ability in preschool children", en Journal of Experimental Child Psychology, 83, 2: 111-130.

Banai K, Ahissar, M. (2013). "Musical Experience, Auditory Perception and Reading-Related Skills in Children", en PLOS ONE 8,9: e75876.

Bhide, A., Power, A., y Goswami, U. (2013). "A Rhythmic Musical Intervention for Poor Readers: A Comparison of Efficacy With a Letter Based Intervention", en Mind, Brain, and Education, 7, 2: 113-123.

Bolduc, J. (2009). "Effects of a music programme on kindergartners' phonological awareness skill 1", en International Journal of Music Education, 27,1: 37-47.

Bolduc, J., y Montésinos-Gelet, I. (2005). "Pitch processing and phonological awareness", en Psychomusicology, 19,1: 3-14.

Butzlaff, R. (2000). "Can music be used to teach reading?", en Journal of Aesthetic Education, 34, 3-4 167-78.

Carmon, Y., Even-Zohar, S., y Wohl, A. (2008). "The Musical Notes Method for Initial Reading Acquisition", en Journal of Cognitive Education and Psychology, 7, 1: 81-100.

Chobert, J., y Besson, M. (2013). "Musical Expertise and Second Language Learning", en Brain Sciences, 3, 2: 923-940.

Cogo-Moreira, H., de Avila, C. R. B., Ploubidis, G. B., y de Jesus Mari, J. (2013). "Effectiveness of Music Education for the Improvement of Reading Skills and Academic Achievement in Young Poor Readers: A Pragmatic Cluster-Randomized, Controlled Clinical Trial”, en PloS one, 8,3: e59984. 
Corrigall, K. A., y Trainor, L. J. (2011). "Associations between length of music training and reading skills in children", en Music Perception, 29,2: 147-155.

David, D., Wade-Woolley, L., Kirby, J. R., y Smithrim, K. (2007). "Rhythm and reading development in school-age children: a longitudinal study", en Journal of Research in Reading, 30,2: 169-183.

Degé, F., y Schwarzer, G. (2011). "The effect of a music program on phonological awareness in preschoolers", en Frontiers in psychology, 2.

Douglas, S., y Willats, P. (1994). "The Relationship between musical ability and literacy skills", en Journal of Research in Reading, 17: 99-107.

Estudio Europeo de Competencia Lingüística. (2012). Ministerio de Educación, Cultura y Deporte e Instituto Nacional de Evaluación Educativa: Secretaria General Técnica.

Disponible en: http://www.mecd.gob.es/dctm/ievaluacion/internacional/eeclvolumeni.pdf?documentId $=0901 \mathrm{e} 72 \mathrm{~b} 813 \mathrm{ac} 515$, consultado 7 de octubre, 2014 .

Fisher, D. (2001). "Early language learning with and without music", en Reading Horizons, 42.1: 39-49.

Forgeard, M., Schlaug, G., Norton, A., Rosam, C., Iyengar, U., y Winner, E. (2008). “The Relation between Music and Phonological Processing in Normal-Reading Children and Children with Dyslexia”, en Music Perception: An Interdisciplinary Journal, 25, 4: 383-390.

Foy, J. G., y Mann, V. (2003). "Home literacy environment and phonological awareness in preschool children: Differential effects for rhyme and phoneme awareness", en Applied Psycholinguistics, 24.1: 59-88.

Gromko, J. E. (2005). "The Effect of Music Instruction on Phonemic Awareness in Beginning Readers", en Journal of Research in Music Education, 53, 3: 199-209.

Hansen, D., y Bernstorf, E. (2002). "Linking music instruction to reading instruction", en Music Educators Journal, 88, 5: 17-21.

Herrera, L., Lorenzo, O., Defior, S., Fernández-Smith, G., y Costa-Giomi, E. (2011). "Effects of phonological and musical training on the Reading readiness of native- and foreign- Spanish- speaking children", en Psychology of Music, 39, 1: 68-81.

Ho,Y., Cheung, M., y Chan, A.S. (2003). "Music training improves verbal but not visual memory: Cross-sectional and longitudinal explorations in children", en Neuropsychology, 17,3: 439-450.

Huss, M., Verney, J. P., Fosker, T., Mead, N., y Goswami, U. (2011). "Music, rhythm, rise time perception and developmental dyslexia: perception of musical meter predicts reading and phonology", en Cortex, 47,6: 674-689.

Karrass, J., y Braungart-Rieker, J. M. (2005). "Effects of shared parent-infant book reading on early language acquisition", en Journal of Applied Developmental Psychology, 26.2: 133-148.

Lamb, S.J., y Gregory, A. H. (1993). "The relationship between music and reading in beginning readers", en Educational Psychology, 13: 19-27.

Lane, H. B., y Wright, T. L. (2007). "Maximizing the effectiveness of reading aloud", en The Reading Teacher, 60.7: 668-675.

Lessard, A., y Bolduc, J. (2011). "Links between Musical Learning and Reading for First to Third Grade Students: A Literature Review”, en International Journal of Humanities and Social Science, $1,7$.

Moreno, S., Marques, C., Santos, A., Santos, M., y Besson, M. (2009). "Musical Training Influences Linguistic Abilities in 8-Year-Old Children: More Evidence for Brain Plasticity", en Cerebral Cortex 19,3: 712-723. 
Moreno, S., Friesen, D., y Bialystok, E. (2011). "Effect of music training on promoting preliteracy skills: preliminary causal evidence", en Music Perception, 29, 2: 165-172.

Moritz, C., Yampolsky, S., Papadelis, G., Thomson, J., y Wolf, M. (2012). "Links between early rhythm skills, musical training, and phonological awareness", en Reading and Writing, 1-31.

National Assessment of Educational Progress (NAEP). (2011). Washington, D.C.: The Nation's Repord Card. National Center for Education Statistics, Institute of Education Sciences, U.S. Department of Education.

Peynircioglu, Z. F., Durgunoglu, A.Y. y Öney-Küssefoglu, B.(2002). "Phonological Awareness and musical aptitude", en Journal of Research in Reading, 25, 1: 68-80.

Phillips, L. M., Norris, S. P., y Anderson, J. (2008). "Unlocking the door: Is parents' reading to children the key to early literacy development?", en Canadian Psychology/Psychologie canadienne, 49.2:82.

Ramón, P. R., y Sánchez, J. N. G. (2009). "El entorno familiar y su influencia en el rendimiento académico de los alumnos con dificultades de aprendizaje: revisión de estudios empíricos", en Aula abierta, 37.1: 117-128.

Register, D. (2001). "The effects of an early intervention music curriculum on prereading/writing", en Journal of Music Therapy, 38,3: 239-248.

Register, D. (2004). "The effects of live music groups versus an educational children's television program on the emergent literacy of young children", en Journal of Music Therapy, 41,1: 2-27.

Register, D., Darrow, A.A., Standley, J., y Swedberg, O. (2007). "The use of Music to enhance reading skills of second grade students and students with reading disabilities", en Journal of Music Theraphy, 44.1: 23-37.

Runfola, M., Etopio, E., Hamlen, K., y Rozendal, M. (2012). "Effect of Music Instruction on Preschoolers' Music Achievement and Emergent Literacy Achievement", en Bulletin of the Council for Research in Music Education, 192: 7-27.

Slevc, L.R., y Miyake, A. (2006). "Individual differences in second language proficiency: Does Musical Ability Matter?", en Psychological Science, 17,8: 675-681.

Standley, J. M. (2008). "Does Music Instruction Help Children Learn To Read?. Evidence of a Meta-Analysis", en Update: Applications of Research in Music Education, 27,1: 17-32.

Taub, G. E., y Lazarus, P. J. (2013). "The effects of training in timing and rhythm on reading achievement", en Contemporary Issues in Education Research (CIER), 5, 4: 343-350.

Tierney, A. T., y Kraus, N. (2013a). "Music training for the development of reading skills", en Applying Brain Plasticity to Advance and Recover Human Ability Progress in Brain Research.

Tierney, A. T., y Kraus, N. (2013b). "The Ability to Move to a Beat Is Linked to the Consistency of Neural Responses to Sound", en The Journal of Neuroscience, 33, 38: 14981-14988.

Toscano-Fuentes, C.M., y Fonseca-Mora, M.C. (2012). "La música como herramienta facilitadora del aprendizaje del inglés como lengua extranjera”, en Teoría de la educación, 24, 2: 197-213.

Tsang, C. D., y Conrad, N. J. (2011). "Music training and reading readiness", en Music Perception, 29, 2: 157-163.

Whalley, K., y Hansen, J. (2006). "The role of prosodic sensitivity in children's reading development", en Journal of Research in Reading, 29,3: 288-303.

Zuk, J., Andrade, P. E., Andrade, O. V., Gardiner, M., y Gaab, N. (2013). "Musical, language, and reading abilities in early Portuguese readers", en Frontiers in Psychology, 4. 